\title{
Novel designed polyoxyethylene nonionic surfactant with improved safety and efficiency for anticancer drug delivery
}

This article was published in the following Dove Press journal:

International Journal of Nanomedicine

28 April 2014

Number of times this article has been viewed

\section{Chang $\mathrm{Li}^{1}$ \\ Chunmeng Sun' \\ Shasha $\mathrm{Li}^{\mathrm{I}}$ \\ Peng $\mathrm{Han}^{2}$ \\ Huimin Sun ${ }^{3}$ \\ Ammar Ouahab' \\ Yan Shen' \\ Yourui $\mathrm{Xu}{ }^{\prime}$ \\ Yerong Xiong' \\ Jiasheng Tu}

'State Key Laboratory of Natural Medicines, Department of

Pharmaceutics, China Pharmaceutical University, Nanjing, ${ }^{2}$ Chinese

Pharmacopoeia Commission, Beijing,

${ }^{3}$ National Institute for Food and Drug

Control, Beijing, People's Republic of

China
Correspondence: Yan Shen; Jiasheng Tu State Key Laboratory of Natural

Medicines, Department of Pharmaceutics, Pharmaceutical University, Nanjing,

People's Republic of China

Tel +86258327 I 305

Email shenyan I982080I@I26.com;

jiashengtu@aliyun.com

\begin{abstract}
In order to limit the adverse reactions caused by polysorbate 80 in Taxotere ${ }^{\circledR}$, a widely used formulation of docetaxel, a safe and effective nanocarrier for this drug has been developed based on micelles formed by a new class of well-defined polyoxyethylene sorbitol oleate (PSO) with sorbitol as the matrix in aqueous solution. The physicochemical properties of the amphiphilic surfactant and the resulting micelles can be easily fine-tuned by the homogeneous sorbitol matrix and pure oleic acid. Composition, critical micelle concentration, and entrapment efficiency were investigated by ultraviolet visible spectroscopy, matrix-assisted laser desorption/ionization time-of-flight mass spectrometry, fluorospectrophotometry, and high-performance liquid chromatography. In vitro and in vivo evaluation revealed that PSO had exceptionally low hemolysis and histamine release rates compared with commercial polysorbate 80 . Moreover, the tumor targeting delivery of PSO was investigated by in vivo imaging in S180 tumor-bearing mice. The results suggest that this novel delivery system, PSO, provides an acceptable alternative to polysorbate 80 for delivery of docetaxel. Further, due to the hypoallergenic nature of PSO, the mechanism of pseudoallergy caused by the polyoxyethylene nonionic surfactant was investigated. Based on in vitro cell analysis, it was assumed that the initial contact of polyoxyethylene nonionic surfactant with mast cells provoked pseudoallergy via polyamine receptor-mediated endocytosis.
\end{abstract}

Keywords: polyoxyethylene nonionic surfactant, sorbitol, isosorbide, pseudoallergy, docetaxel

\section{Introduction}

Improving delivery of hydrophobic anticancer drugs to target tissue is one of the most difficult challenges facing the pharmaceutical industry. A wide variety of nanocarriers has been used to overcome this problem. ${ }^{1}$ Karve et al demonstrated that nanoparticle drug delivery systems could promote the readoption of abandoned drugs by overcoming drug delivery challenges ${ }^{2}$ because of their unique properties, such as preferential accumulation in tumor tissue and reduced distribution in normal tissues. Current research is focused on developing nanoparticle formulations of widely used chemotherapeutics or newly discovered cancer agents, which are few in number. ${ }^{3}$ Traditional self-assembled polyoxyethylene nonionic surfactants (PNS), including polysorbate 80, polyoxyl 35 castor oil, poloxamer, and poly(ethylene glycol), are a class of nanocarrier systems with potential application in tablets, ${ }^{4}$ emulsions, ${ }^{5}$ and especially in the preparation of injections. ${ }^{6}$ PNS can dramatically improve the efficiency of anticancer drugs by enhancing their solubility in aqueous solution. However, due to the different synthetic processes used by different manufacturers to obtain PNS products, their 
structure and composition are not identical from batch to batch. Hence, no structural formula for PNS is available in any pharmacopeia. For example, in the United States Pharmacopeia 35-National Formulary 30, polysorbate 80 was defined as a mixture of partial esters of fatty acids, mainly oleic acid, with sorbitol and its anhydrides ethoxylated with approximately 20 moles of ethylene oxide for each mole of sorbitol and sorbitol anhydrides. ${ }^{7}$ Diverse manufacturing procedures for polysorbate 80 have been reported. ${ }^{8}$ Briefly, one method involves dehydration of sorbitol to its derivative, followed by esterification with oleic acid and polyreaction of ethylene oxide, and another method is conducted by polyreaction followed by esterification. The widely accepted structure of polysorbate 80 is shown in Figure 1A. To our knowledge, the first step in the synthesis of polysorbate is dehydration of sorbitol to sorbitan, suggesting that the final product is a mixture of sorbitol and sorbitol-derived cyclic ethers with different structures (Figure 1B).

Furthermore, the potential of PNS to trigger pseudoallergy is well known. ${ }^{9-11}$ Pseudoallergy is the official term used by the World Allergy Organization. It is a reaction similar to an immune allergic reaction that is observed following the first administration of the offending agent. Unlike common allergies, pseudoallergic reactions can directly induce release of histamine from mast cells and activate the complement system, with abnormal synthesis of eicosanoids and inhibition of bradykinin degradation, which are not initiated or mediated by pre-existing immunoglobulin E antibodies. ${ }^{12}$ Although the exact mechanisms of pseudoallergy in response

A

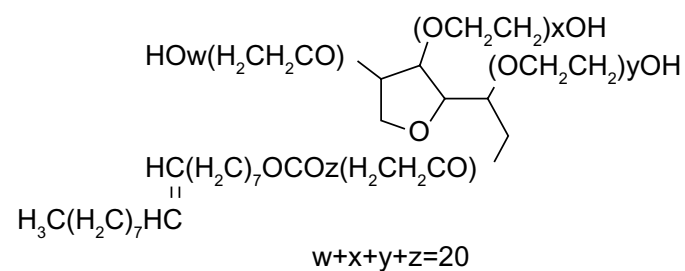

B

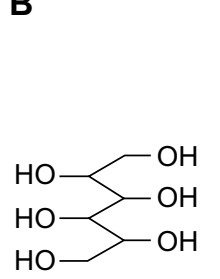

Sorbitol

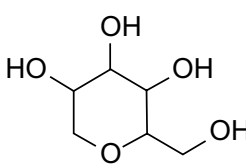

1,5-sorbitan

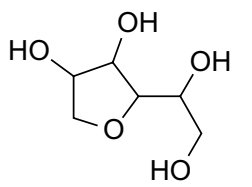

1,4-sorbitan

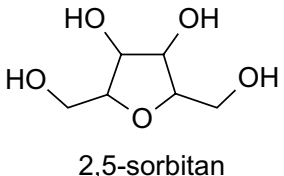

2,5-sorbitan

$\mathrm{HO}$

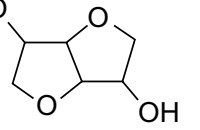

1,4:3,6-isosorbide
Figure I Typical chemical structures of polysorbate 80 (A), and sorbitol and sorbitol-derived cyclic ethers (B). to PNS remain unclear, it is believed that activation of the complement system and degranulation of mast cells initiate the reactions that result in pseudoallergy, ie, the initial step of the pseudoallergic reaction is the key step.

In recent decades, product impurities, such as peroxide and oxirane, ${ }^{13,14}$ along with residual fatty acids that are not completely consumed in the reaction, have been considered to be the major triggers of pseudoallergies, but early on in the research in this area it was found that the histamine release rate was not reduced even when using a highly purified fatty acid with strictly controlled impurities. Therefore, we focused on the structures of the PNS themselves in an attempt to determine the structure-activity relationship between PNS and their safety.

In the present investigation, polyoxyethylene sorbitol oleate (PSO), based on polysorbate 80 containing a homogenous sorbitol matrix, was developed for safer and more effective delivery of docetaxel, an important hydrophobic anticancer drug. We selected the RBL-2H3 mast cell line to study secretion of the anaphylactic mediator in vitro, given that many investigators had used this cell line in early studies. Because of the difficulties involved in measuring amounts of histamine in vitro and the lack of consistent results, $\beta$-hexosaminidase activity is generally used to monitor histamine release and mast cell degranulation and to investigate the mechanisms of pseudoallergy. ${ }^{15}$

\section{Methods and materials}

\section{Materials and animals}

Commercial polysorbate 80 was purchased from different manufacturers. PSO and polyoxyethylene isosorbide oleate (PIO) were synthesized and supplied by Will Chemical Co, Ltd (Nanjing, People's Republic of China). RBL-2H3 mast cell lines were purchased from the Shanghai Institutes for Biological Sciences, Chinese Academy of Sciences (Shanghai, People's Republic of China). Compound 48/80 was purchased from Sigma-Aldrich (St Louis, MO, USA) and kept at $-20^{\circ} \mathrm{C}$ away from light. p-Nitrophenyl-2-acetamido2 -deoxy- $\beta$-D-glucopyranoside was sourced from Feiyang Biotechnology Co, Ltd (Shandong, People's Republic of China). MTT (3-[4,5-dimethylthiazol-2-yl]-2,5 diphenyl tetrazolium bromide) was purchased from Biosharp (Seoul, Republic of Korea). Dimethylsulfoxide was obtained from Jiuyi Chemical Co, Ltd (Shanghai, People's Republic of China). Triton X-100 was purchased from Amresco (Solon, OH, USA). Benzalkonium chloride (BAC) was supplied by Robiot Co Ltd (Nanjing, People's Republic of China). 2-deoxy-D-glucose was sourced from Aladdin 
(Los Angeles, CA, USA). DiR (1,1'-dioctadecyl-3,3,3',3'tetramethylindotricarbocyanine iodide) was obtained from Biotium Inc. (Hayward, CA, USA). All other reagents were chromatographic grade.

Guinea pigs and New Zealand White rabbits were purchased from Qinglong Mountain Farm, Nanjing, People's Republic of China. All animal experiments were implemented according to the National Institute of Health Guide for the Care and Use of Laboratory Animals and approved by the animal ethics committee of China Pharmaceutical University.

\section{Cell culture}

RBL-2H3 cells were maintained as a monolayer culture in Eagle's Minimum Essential Medium (HyClone Laboratories; Logan, UT, USA) with $15 \%$ fetal bovine serum and $1 \%$ penicillin/streptomycin in an atmosphere of $5 \% \mathrm{CO}_{2}$ at $37^{\circ} \mathrm{C}$.

\section{Ultraviolet-visible absorption spectra of commercial polysorbate $80, \mathrm{PSO}$, and PIO}

Test samples were dissolved in a solution of acetonitrile/ water $(70 / 30, \mathrm{v} / \mathrm{v})$ then diluted to a concentration of $4 \mathrm{mg} / \mathrm{mL}$ for the test solutions. The test solutions were scanned at varying wavelengths ranging from $190 \mathrm{~nm}$ to $400 \mathrm{~nm}$ using a UV 3200 spectrophotometer (Mapada Instruments, Shanghai, People's Republic of China). ${ }^{16}$

\section{MALDI-TOF MS assay}

A matrix-assisted laser desorption/ionization time-offlight (MALDI TOF) mass spectrophotometer (Shimadzu, Tokyo, Japan) was used to analyze the molecular weight distribution of PSO and PIO. First, $30 \mu \mathrm{L}$ samples were dispersed in $200 \mu \mathrm{L}$ of methanol as the analyte solution. A saturated solution of $\mathrm{CH}_{3} \mathrm{CN} / \mathrm{H}_{2} \mathrm{O} / \mathrm{TFA}(50 / 50 / 0.1, \mathrm{v} / \mathrm{v} / \mathrm{v})$ with $\alpha$-cyano-4-hydroxycinnamic acid was then prepared as the matrix solution. Next, $1 \mu \mathrm{L}$ of both solutions was mixed to make the test solution before analysis, and a small volume of the mixture was loaded on a sample plate then dried spontaneously. The dried sample was analyzed using the MALDI-TOF mass spectrophotometer equipped with a MALDI ion source. ${ }^{17}$

\section{Matrix analysis of PSO and PIO}

Each sample was refluxed with $2 \%$ methylated $\mathrm{NaOH}$ in a water bath at $80^{\circ} \mathrm{C}$. After 35 minutes of reflux, $50 \mathrm{~mL}$ of water was added to the reaction. The solutions were acidified with $5 \% \mathrm{HCl}$ and cooled to room temperature. The substances in each solution were extracted with $20 \mathrm{~mL}$ of heptane. The aqueous layer was collected as the test solution, ${ }^{8}$ then determined by high-performance liquid chromatography (HPLC) equipped with an LC-20AT pump and RID-10A refractive index detector (Shimadzu). The injection volume was $20 \mu \mathrm{L}$ and the mobile phase was $0.05 \%$ sulfuric acid aqueous solution eluted at a flow rate of $0.60 \mathrm{~mL}$ per minute. All separations were carried out on an Aminex HPX-87H ion exclusion column $(7.8 \times 300 \mathrm{~nm}$, Bio-Rad, Hercules, CA, USA) at $55^{\circ} \mathrm{C}$.

\section{In vitro studies of PSO and PIO Cytotoxicity}

RBL-2H3 cells were used for the cytotoxicity studies of PSO and PIO. Cells were seeded in 96-well plates (approximately $1 \times 10^{5}$ cells/well) and grown in Roswell Park Memorial Institute 1640 medium (HyClone) containing 10\% bovine calf serum at $37^{\circ} \mathrm{C}$ for 24 hours in a $95 \%$ humidity and $5 \% \mathrm{CO}_{2}$ atmosphere. When cells reached confluence, the medium was replaced with $100 \mu \mathrm{L}$ Roswell Park Memorial Institute 1640 medium containing $1 \%$ and 3\% PSO or PIO. After incubation for 24 hours, $20 \mu \mathrm{L}$ MTT $(5 \mathrm{mg} / \mathrm{mL})$ was added to each well followed by incubation for a further 4 hours. The medium was then replaced with $200 \mu \mathrm{L}$ of dimethylsulfoxide. Following agitation for 10 minutes, viable cells were quantified by recording the ultraviolet absorbance at $490 \mathrm{~nm}$ using a microplate reader (Bio-Tek Instruments, Winooski, VT, USA). ${ }^{18}$

\section{$\beta$-hexosaminidase release assay}

The $\beta$-hexosaminidase release assay was performed as described elsewhere. ${ }^{15}$ In brief, confluent RBL-2H3 cells were seeded in a 96-well plate. Cells attached to the microtiter wells were then washed twice with Tyrode's buffer and stimulation was initiated by adding $200 \mu \mathrm{L}$ of Tyrode's buffer containing PSO, PIO, or commercial polysorbate $80(1 \%, w / w)$. After one hour of incubation at $37^{\circ} \mathrm{C}$, degranulation was terminated by placing the plates in ice. To determine the amount of $\beta$-hexosaminidase released from the cells, $60 \mu \mathrm{L}$ of supernatant and $60 \mu \mathrm{L}$ of $\beta$-hexosaminidase substrate solution $(5 \mathrm{mmol} / \mathrm{mL}$ p-nitrophenyl-2-acetamido-2-deoxybeta-D-glucopyranoside in $0.1 \mathrm{~mol} / \mathrm{mL}$ sodium acetate buffer, $\mathrm{pH} 4.5$ ) were mixed in a new 96-well plate and incubated for one hour at $37^{\circ} \mathrm{C}$. Next, $150 \mu \mathrm{L}$ of carbonate buffer containing $100 \mathrm{mmol} / \mathrm{L} \mathrm{NaCO}_{3}$ and $100 \mathrm{mmol} / \mathrm{L} \mathrm{NaHCO}_{3}$ buffer at $\mathrm{pH} 10.7$ was added to the wells to quench the reaction. A negative control group incubated with Tyrode's buffer only was used to assess spontaneous release of $\beta$-hexosaminidase. The total amount of $\beta$-hexosaminidase present was obtained by lysing the cells with $0.1 \%$ Triton X-100 prior to removal 
of the supernatant. $\beta$-hexosaminidase in the supernatant was quantified by measuring the absorbance at $450 \mathrm{~nm}$ using an ELx800 microplate reader (Bio-Tek Instruments). The background absorbance of substrate in Tyrode's buffer alone (no cell supernatant) was subtracted from all readings.

\section{Hemolysis}

Hemolysis was investigated using a method described previously. ${ }^{19}$ Blood was collected from anesthetized New Zealand White rabbits by cardiac puncture. Whole blood was centrifuged at 3,000 rpm for 10 minutes, and the isolated red blood cells were washed with physiological saline. The red blood cells were then suspended in physiological saline to obtain a concentration of $2 \%$. Aliquots of the $2 \%$ red blood cell suspension containing the tested carriers at different concentrations in the range of $2-20 \mathrm{mg} / \mathrm{mL}$ were incubated at $37^{\circ} \mathrm{C}$ for one hour. The positive and negative controls were water and physiological saline, respectively. The samples were then centrifuged at 3,000 rpm for $10 \mathrm{~min}-$ utes to remove nonlysed red blood cells. The supernatant was collected and analyzed using a microplate reader (Bio-Tek Instruments) at $540 \mathrm{~nm}$. The hemolysis rate was determined using the following formula:

$$
\text { Hemolysis }(\%)=\frac{A_{\text {sample }}-A_{\text {negative }}}{A_{\text {positive }}-A_{\text {negative }}} \times 100 \text {. }
$$

\section{In vivo imaging}

Approximately $10^{7} \mathrm{~S} 180$ cells were inoculated subcutaneously in the flank region of mice. When the tumor volume reached about $700 \mathrm{~mm}^{3}$, the mice were injected with DiRloaded commercial polysorbate 80 or PSO $(200 \mu \mathrm{g} / \mathrm{kg})$ via the tail vein and then anesthetized using an intramuscular injection of chloral hydrate $(20 \mathrm{mg} / \mathrm{kg})$. In vivo imaging experiments were performed at 30 minutes and 2, 8, and 12 hours following injection using a multimodal IS2000MM imaging system (Kodak, Rochester, NY, USA) equipped with an excitation band pass filter at $720 \mathrm{~nm}$ and an emission at $790 \mathrm{~nm}$. Images were analyzed using the IS2000MM imaging station software (Kodak ID Image Analysis Software; Kodak). ${ }^{20,21}$

\section{Critical attributes of PSO}

\section{Critical micelle concentration}

The critical micelle concentration was determined using a method reported previously. ${ }^{22}$ Briefly, pyrene was used as the fluorescence probe. An aliquot of $0.2 \mathrm{~mL}$ pyrene solution $(4.8 \mu \mathrm{g} / \mathrm{mL})$ in acetone was added to a series of volumetric flasks, and the acetone was then evaporated to dryness under a nitrogen stream. A series of concentrations of PSO solution was added and equilibrated at room temperature in a lightproof water bath for 24 hours before measurement. The excitation spectrum of pyrene was determined using an RF-5301 PC fluorescence spectrophotometer (Shimadzu). The intensity ratio $\mathrm{I}_{338} / \mathrm{I}_{333}$ was calculated and plotted against the logarithm of PSO mass concentration. Critical micelle concentration was indicated by the inflection in the curve.

\section{Entrapment efficiency}

First, docetaxel $1 \mathrm{mg} / \mathrm{mL}$ in ethanol was mixed with $10 \mathrm{mg} / \mathrm{mL}$ PSO in ethanol or polysorbate 80 in ethanol at a series of weight ratios of 1:5, 1:15, and 1:25. Ethanol was then removed under vacuum, and the mixtures were dissolved in $10 \mathrm{~mL}$ of double-distilled water. After centrifugation (at 3,000 rpm for 10 minutes), the supernatant was removed and analyzed by HPLC to determine the docetaxel loading. The mobile phase consisted of a series of concentrations of acetonitrile/water solution (shown in Table 1) flowing at a rate of $1.2 \mathrm{~mL}$ per minute. All separations were carried out on a $\mathrm{C}_{18}$ column $(4.6 \times 150 \mathrm{~mm}, 3.5 \mu \mathrm{m}$, Waters Corporation, Milford, MA, USA) maintained at $45^{\circ} \mathrm{C}$ with a detection wavelength of $232 \mathrm{~nm}$. The injection volume of the samples was $20 \mu \mathrm{L}$. The entrapment efficiency (EE) was calculated as follows:

$$
\text { EE }(\%)=\frac{\text { Weight of docetaxel in micelles }}{\text { Weight of docetaxel fed initially }} \times 100 \text {. }
$$

\section{In vivo study of pseudoallergy triggered by PSO, PIO, and commercial products}

Guinea pigs (weight 250-300 g, 12 male and 12 female) were randomly divided into six groups with four animals in each group. Group 1 was treated with physiological saline (negative control), group 2 was treated with $0.15 \mathrm{mg} / \mathrm{mL}$ ovalbumin solution (positive control), group 3 was treated with $10 \%$ PSO solution, group 4 was treated with $10 \%$ PIO solution, group 5 was treated with $10 \%$ commercial polysorbate $80-1$, and group 6 was treated with $10 \%$ commercial

Table I Gradient elution

\begin{tabular}{lll}
\hline Time (minutes) & Water (\%) & Acetonitrile (\%) \\
\hline 0.0 & 72 & 28 \\
9.0 & 72 & 28 \\
39.0 & 28 & 72 \\
39.1 & 72 & 28 \\
50.0 & 72 & 28 \\
\hline
\end{tabular}


polysorbate 80-2. Each group received a dose of $2 \mathrm{~mL} / \mathrm{kg}$ intravenously. The responses of the guinea pigs were observed and recorded 30 minutes after injection. ${ }^{23}$ Symptoms of a systemic sensitization reaction and their respective severity are listed in Table 2.

\section{Mechanism of PNS-triggered mast cell degranulation}

Most nanocarriers used for injection can trigger mast cell degranulation followed by histamine release and pseudoallergy. However, the exact structure-activity relationship is not clearly understood. Previous data have shown that BAC is a selective inhibitor of compound $48 / 80$ and the polyamine receptor, ${ }^{24}$ and release of histamine can also be significantly suppressed at low temperatures $\left(4^{\circ} \mathrm{C}\right) .{ }^{25}$

\section{Effect of selective inhibition of the polyamine receptor on PNS-induced $\beta$-hexosaminidase release} An appropriate amount of BAC was dissolved in solutions containing PSO, PIO, or commercial polysorbate 80 , respectively, and then diluted to give final BAC concentrations of $1.5 \mu \mathrm{g} / \mathrm{mL}$ and $3 \mu \mathrm{g} / \mathrm{mL} .{ }^{24} \mathrm{~A} \beta$-hexosaminidase release assay was done as described in the $\beta$-hexosaminidase release assay section.

\section{Temperature-dependence and energy-dependence of PNS-induced $\beta$-hexosaminidase release}

A $\beta$-hexosaminidase release assay was performed following the procedures mentioned in the $\beta$-hexosaminidase release assay section except that the temperature was set at $4^{\circ} \mathrm{C}$ instead of $37^{\circ} \mathrm{C}$ in order to investigate the effect of low temperature. ${ }^{25}$ The RBL-2H3 cells were preincubated with 2-deoxy-D-glucose for one hour to exhaust the energy in the energy-dependent experiment. ${ }^{26}$

\section{Statistical analysis}

The data are expressed as the mean \pm standard deviation of triplicate experiments performed in a parallel manner unless

Table 2 Symptoms of allergic reaction in vivo

\begin{tabular}{lll}
\hline $\begin{array}{l}\text { Allergy } \\
\text { strength }\end{array}$ & Symptoms & Level \\
\hline- & Normal & \\
+ & Piloerection, shivering, itchy nose & Negative \\
++ & Sneezing, cough, micturition, defecation negative & Positive \\
+++ & Dyspnea, wheezing rales, floppy gait, & Strongly positive \\
& spasm, Cheyne-Stokes breathing & \\
++++ & Death & Very strongly \\
& & positive \\
\hline
\end{tabular}

otherwise indicated. The results were analyzed using an unpaired two-tailed Student's $t$-test. All comparisons were made relative to corresponding controls and a statistically significant difference is indicated by $P<0.001, P<0.01$, or $P<0.05$.

\section{Results and discussion Ultraviolet-visible spectra for commercial polysorbate $80, \mathrm{PSO}$, and $\mathrm{PIO}$}

In the present study, ultraviolet-visible spectra $(0.4 \% \mathrm{w} / \mathrm{w})$ for PSO, PIO, and eight randomly numbered commercial formulations of polysorbate 80 from different manufacturers were recorded. These spectra are shown in Figure 2A-C.

Figure $2 \mathrm{~A}$ shows that the spectra of polysorbate 80 are in accordance with results reported previously. ${ }^{16}$ Basically, intense ultraviolet absorbance at $\lambda>250 \mathrm{~nm}$ could be observed due to the presence of residual impurities from the impure fatty acid used in the preparation of polysorbate 80 . To prepare PSO and PIO, fatty acid with high purity ( $>98 \%)$ was used in the reaction, resulting in the only sharp absorbance peak appearing at a wavelength of around $200 \mathrm{~nm}$. The high modifiability of the $\varepsilon$ values for commercial polysorbate 80 at $\lambda>210 \mathrm{~nm}$ suggests that these changes are not due to the core molecular structures, but rather to impurities. Furthermore, polysorbate 80 is a mixture of highly saturated molecules with an alkyl ester group $\left(\lambda_{\max }\left[\mathrm{n} \rightarrow \pi^{*}\right] \sim 195-210 \mathrm{~nm}\right)$, poly(ethylene glycol) chains $\left(\lambda_{\max }\left[\mathrm{n} \rightarrow \pi^{*}\right] \sim 180-185 \mathrm{~nm}\right.$ ) and the only carbon-carbon double bond in the fatty acid chain $\left(\lambda_{\max }\left[\pi \rightarrow \pi^{*}\right] \sim 195 \mathrm{~nm}\right)$. Our results are also consistent with the theoretical values.

\section{MALDI-TOF MS assay}

Given that the MALDI-TOF mass spectrometry assay of commercial polysorbate 80 has already been reported, ${ }^{27}$ commercial polysorbate 80 was not investigated in the present study. The results of MALDI-TOF mass spectrometric analysis of PIO and PSO are shown in Figure 2D and E. The MALDITOF mass spectrum for commercial polysorbate 80 showed two bell-shaped molecular peaks. The commercial samples were composed of sorbitan polyethoxylate, isosorbide polyethoxylate, and free poly(ethylene glycol), ${ }^{17,27}$ but only one bell-shaped peak appeared in each spectra for PIO and PSO, and the peaks are in accordance with the theoretical molecular weight distribution of polysorbate 80 . The highest mass peaks $(\mathrm{m} / \mathrm{z})$ for PIO and PSO were 1,005.9 and 2,232.6, indicating that the major components of the two specially prepared products had a main molecular weight of 1,005.9 and 2,232.6, respectively. However, in the particular case of the peak at $917.8 \mathrm{~m} / \mathrm{z}$, there were two possible chemical structures that have the same molecular weight with different repeat units, ie, 


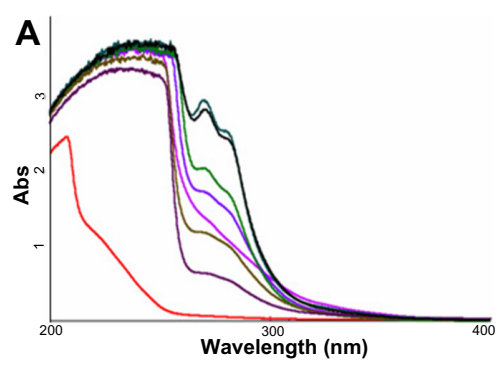

D

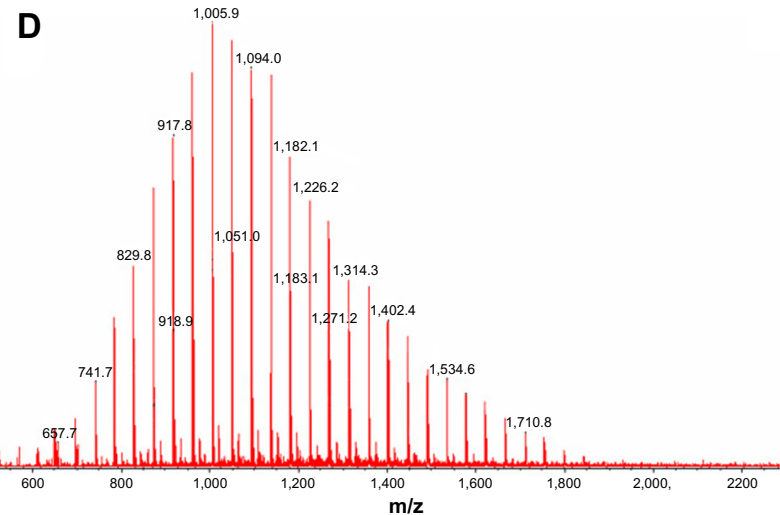

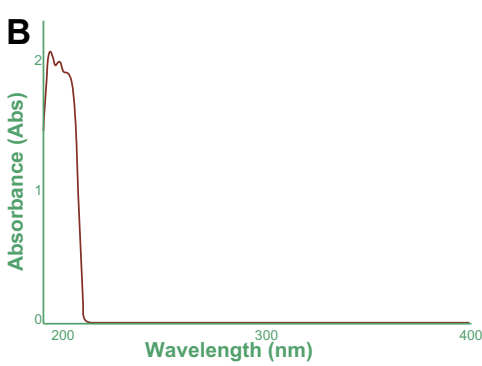

$\mathbf{E}$

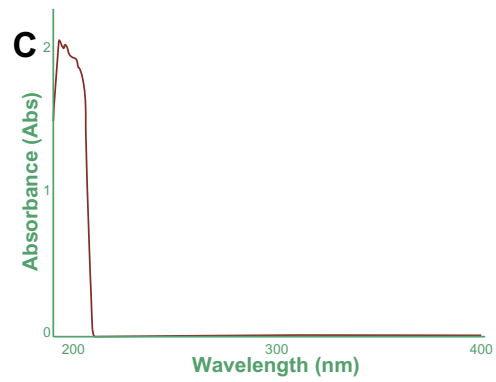

$2,056.4^{2,232.6}$

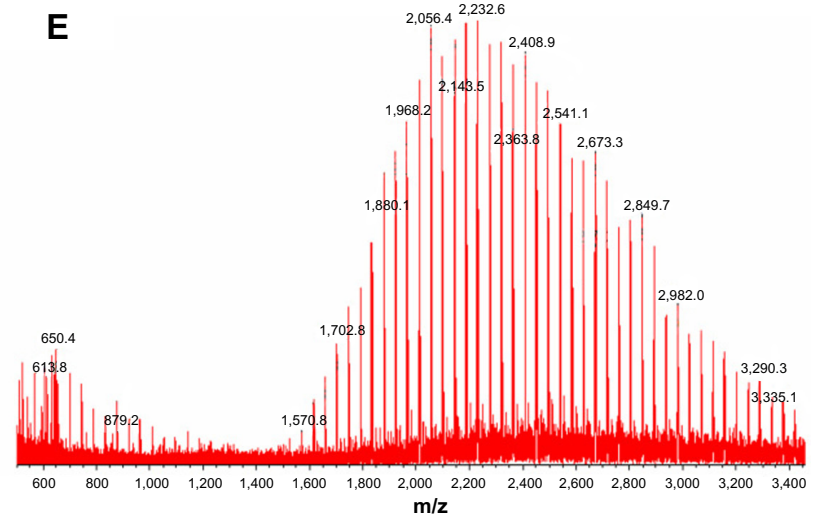

Figure 2 Ultraviolet-visible spectra of eight commercial products (A), polyoxyethylene sorbitol oleate (B) and polyoxyethylene isosorbide oleate (C), and matrix-assisted laser desorption/ionization time-of-flight mass spectra for polyoxyethylene isosorbide oleate (D) and polyoxyethylene sorbitol oleate (E).

Abbreviation: Abs, absorbance units.

eleven repeats of isosorbide polyethoxylate monolinoleate ester and 14 repeats of poly(ethylene glycol) monolinoleate ester. ${ }^{17}$ Although MALDI-TOF mass spectrometry could only analyze the molecular weight distribution of the samples, we still found that the two bell-shaped molecular peaks in the spectra of the commercial products were a combination of the peaks in the spectra of PIO and PSO, suggesting that PIO and PSO were successfully synthesized with a di-dehydration cyclic ester and sorbitol as their core matrix.

\section{Matrix analysis of PSO and PIO}

To further confirm the matrix of PSO and PIO, samples were saponified to remove the fatty acid chains. The residues consisted of the matrix with polyoxyethylene. Separations were achieved by HPLC and are shown in Figure 3.

The retention times for the sorbitol and isosorbide matrices were around 8 minutes and 17 minutes, respectively. From the typical chemical structure of polysorbate 80 (Figure 1A), it is usually considered that 20 moles of ethylene oxide could be polymerized with one mole of sorbitol-derived matrix, regardless of whether it contains sorbitan or isosorbide, but the numbers of ethoxylates are actually inconsistent between PSO and $\mathrm{PIO}$, resulting in a relatively high polydispersity index on analysis by HPLC. Figure $3 \mathrm{~A}$ and $\mathrm{B}$ shows that the only matrix peaks appeared at 8.6 minutes and 17.8 minutes, respectively, indicating that the well-defined matrices of PSO and PIO were sorbitol and isosorbide, respectively. To emphasize the original structures, the whole molecular structure of PSO and PIO were drawn in accordance with original design through HPLC and MALDI-TOF, in Figure $3 \mathrm{~A}$ and B. In contrast, two or more matrices were detected in the saponified commercial polysorbate 80 (Figure $3 \mathrm{C}$ and $\mathrm{D}$ ), demonstrating that PSO and PIO were successfully synthesized as intended.

\section{In vitro studies of PSO and PIO Cytotoxicity}

As shown in Figure 4A, the cytotoxicity of PIO was higher than that of PSO, but not significantly so. The cellular morphology of mast cells after 24 hours of incubation with PSO or $\mathrm{PIO}$ is shown in Figure $4 \mathrm{C}-\mathrm{F}$.

Figure 4B shows that viable mast cells kept their polygonal-shaped morphology and grew adhesively, while it was difficult to find cells with normal morphology in the $3 \%$ concentration group (Figure $4 \mathrm{E}$ and $\mathrm{F}$ ), in which the cells were spherical and formed aggregates. Moreover, our results show that degranulation was not caused by PSO-induced or PIO-induced apoptosis of mast cells.

\section{$\beta$-hexosaminidase release assay}

To evaluate PNS-induced pseudoallergy in vitro, the amounts of $\beta$-hexosaminidase released from RBL-2H3 cells after treatment with PSO, PIO, and commercial 


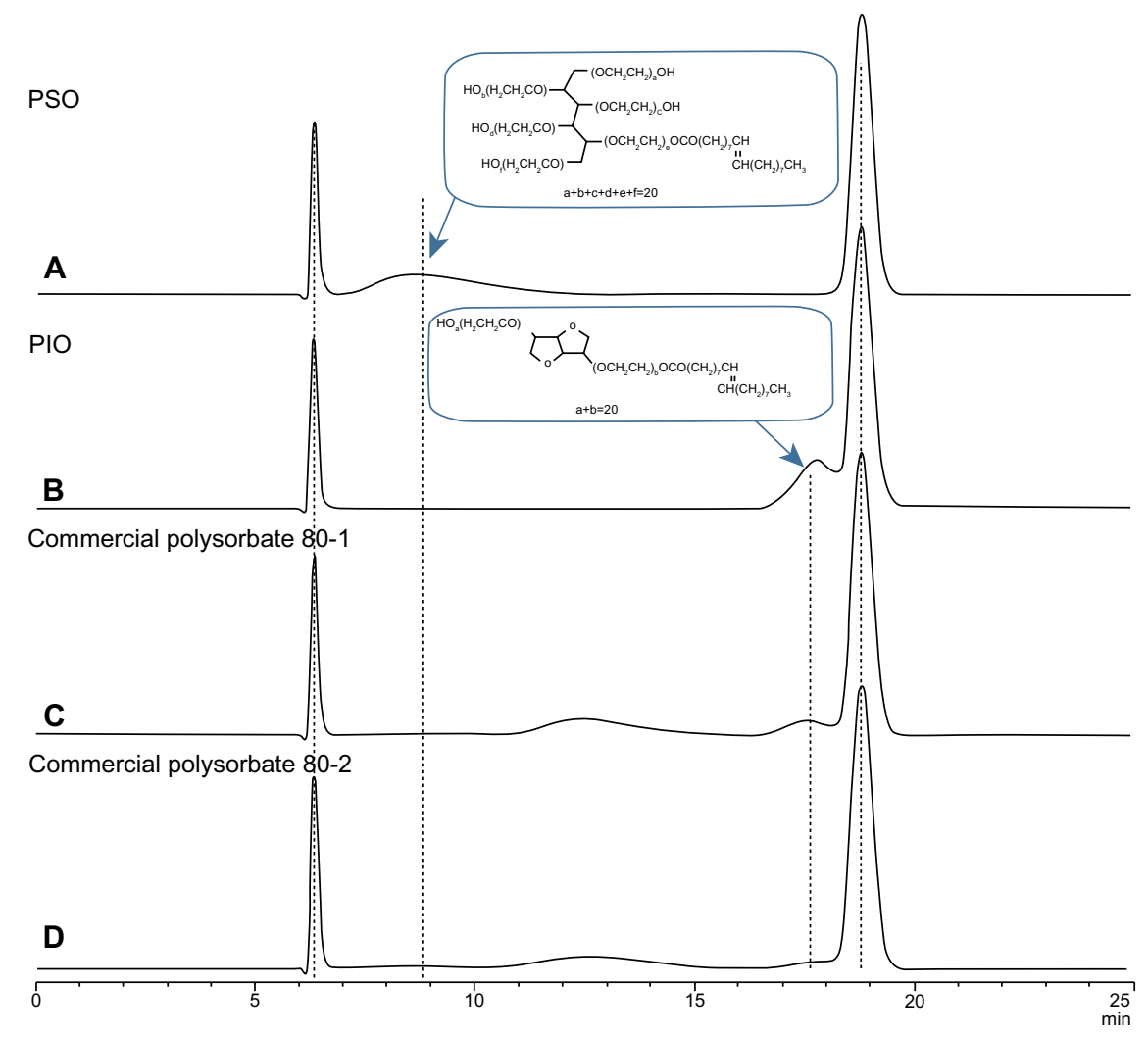

Figure 3 High-performance liquid chromatograms of saponified PSO (A), PIO (B), commercial polysorbate 80-I (C), and commercial polysorbate 80-2 (D). Abbreviations: PSO, polyoxyethylene sorbitol oleate; PIO, polyoxyethylene isosorbide.
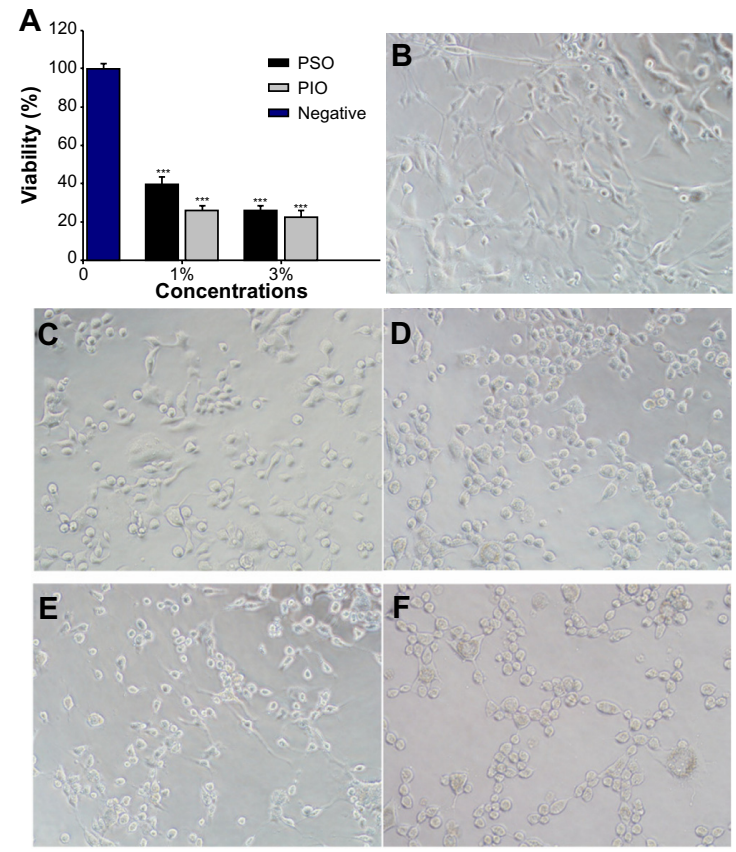

Figure 4 (A) In vitro cytotoxicity of PSO and PIO. The data are shown as the mean \pm standard deviation $(n=5)$. $* * * P<0.001$ versus negative control. (B) Photographs of normal mast cells. (C) Cells after incubation with $1 \%$ PSO for 24 hours. (D) Cells after incubation with I\% PIO for 24 hours. (E) Cells after incubation with 3\% PSO for 24 hours. (F) Cells after incubation with $3 \%$ PIO for 24 hours.

Abbreviations: PSO, polyoxyethylene sorbitol oleate; PIO, polyoxyethylene isosorbide. polysorbate 80 were determined and are shown in Figure $5 \mathrm{~A}$. The results indicate that PIO, commercial polysorbate 80-1, and commercial polysorbate $80-2$ had a similar effect on degranulation of RBL-2H3 cells, and further resulted in release of $\beta$-hexosaminidase. Because PIO is the common ingredient in commercial polysorbate $80-1$ and $80-2$ (Figure $3 \mathrm{C}$ and D), it was postulated that PIO could be one of the potential triggers. However, the lowest $\beta$-hexosaminidase release rate was detected in the PSOtreated group when compared with the other groups, indicating that sorbitol-based PSO had the lowest allergenicity among the samples. In recent decades, product impurities such as dioxane and oxirane along with residual fatty acids that are not completely consumed in reactions have been considered to be the major triggers of pseudoallergy. In this study, PSO and PIO were produced using highly pure fatty acid (>98\%). Therefore, we believe that the difference in $\beta$-hexosaminidase release rate for PSO and PIO is attributable to their core matrix, and PIO with isosorbide as the matrix may be the cause of polysorbate 80 -induced pseudoallergy.

To evaluate PNS-triggered pseudoallergy further at various solution compositions, the $\beta$-hexosaminidase release 
A

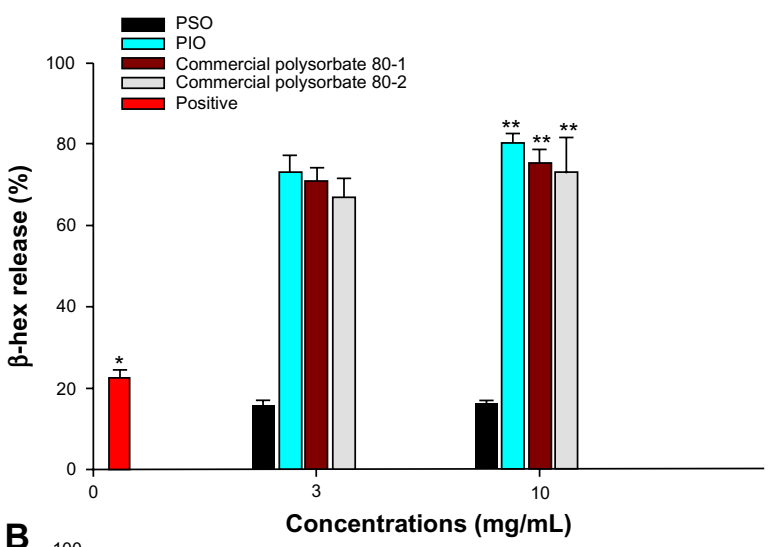

B

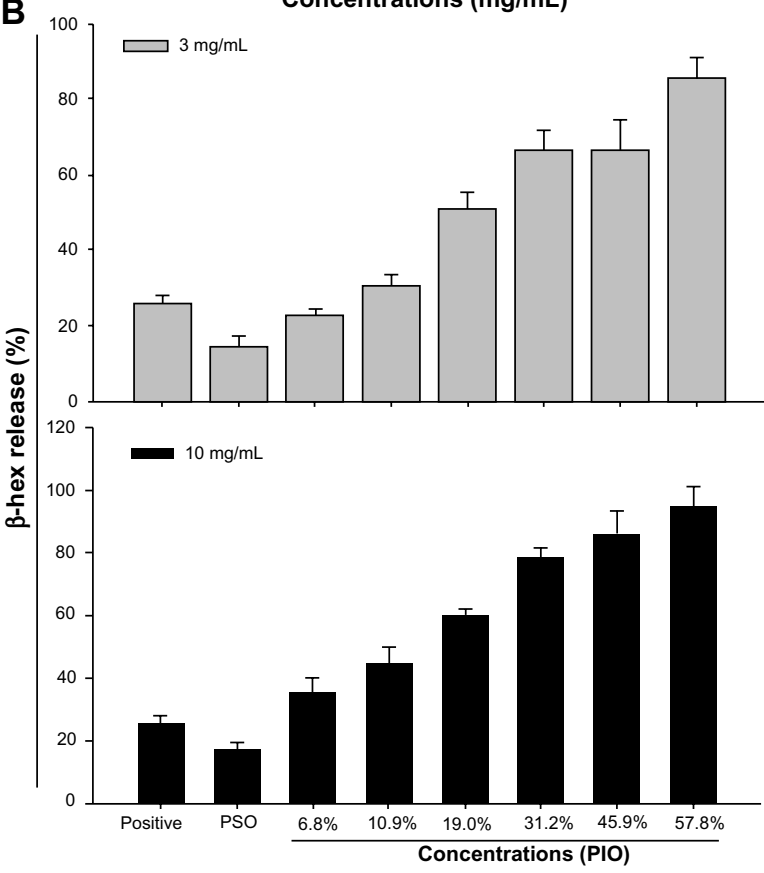

Figure 5 (A) Release rate of $\beta$-hexosaminidase from RBL-2H3 cells after incubation with different samples at $3 \mathrm{mg} / \mathrm{mL}$ and $10 \mathrm{mg} / \mathrm{mL}$. The data are shown as the mean \pm standard deviation $(n=5)$. $* P<0.05$, $* * P<0.01$ versus PSO. (B) PIO-induced $\beta$-hexosaminidase release in a concentration-dependent manner. The data are shown as the mean \pm standard deviation $(n=5)$.

Abbreviations: PSO, polyoxyethylene sorbitol oleate; PIO, polyoxyethylene isosorbide.

assay was performed using a mixture of PIO and PSO at ratios varying from $3 \mathrm{mg} / \mathrm{mL}$ to $10 \mathrm{mg} / \mathrm{mL}$ on the basis of total associated polysorbate 80. As shown in Figure 5B, the $\beta$-hexosaminidase release rate was increased at each concentration point, while the ratio of PIO increased from $0 \%$ to $60 \%$, indicating that commercial polysorbate 80 with a high PIO content would have a high risk of pseudoallergy. However, according to the definition mentioned in the United States Pharmacopeia 35-National Formulary 30, PIO is one of the components of polysorbate 80 , so we believe a useful approach to limiting the allergenicity of polysorbate 80 would be to decrease the PIO content.

\section{Hemolysis study}

As indicated in Figure 6, all groups treated with PIO showed a considerable amount of erythrocyte lysis, whereas PSO caused minimum lysis even at high concentrations of $20 \mathrm{mg} / \mathrm{mL}$. Gill et $\mathrm{al}^{28}$ reported that this hemolytic effect could be used to evaluate the damage done to membranes by nanocarriers. This implies that PSO has less ability to cause membrane damage than PIO. This phenomenon could be explained on the basis that PIO contains isosorbide and has two hydroxyl groups that can easily form diesters with two molecular fatty acids and become more lipophilic, resulting in stronger attachment to the lipid bilayer of erythrocytes and therefore causing destruction of the cell membrane. Our results indicate that PSO, a novel PNS nanocarrier for docetaxel, is not only hypoallergic but also hypohemolytic.

\section{In vivo imaging study}

Mice inoculated with S180 cells were used to investigate the tumor-targeting ability of PSO. Representative fluorescence images after administration of $\mathrm{PSO}$ and commercial polysorbate 80 are shown in Figure 7A and B, respectively. The fluorescent nanocarriers initially spread throughout the whole body after injection, with increased accumulation in tumor tissues after about 2 hours. Bright fluorescence signals of PSO and commercial polysorbate 80 were detected at 12 hours following injection, but the intensity of the fluorescence signal from $\mathrm{PSO}$ was much stronger than that of commercial polysorbate 80 . The results show that the tumor-targeting ability of PSO was not only better than that of commercial polysorbate 80 , but also showed a longer retention time in tumor tissue. PSO could have accumulated at the tumor site

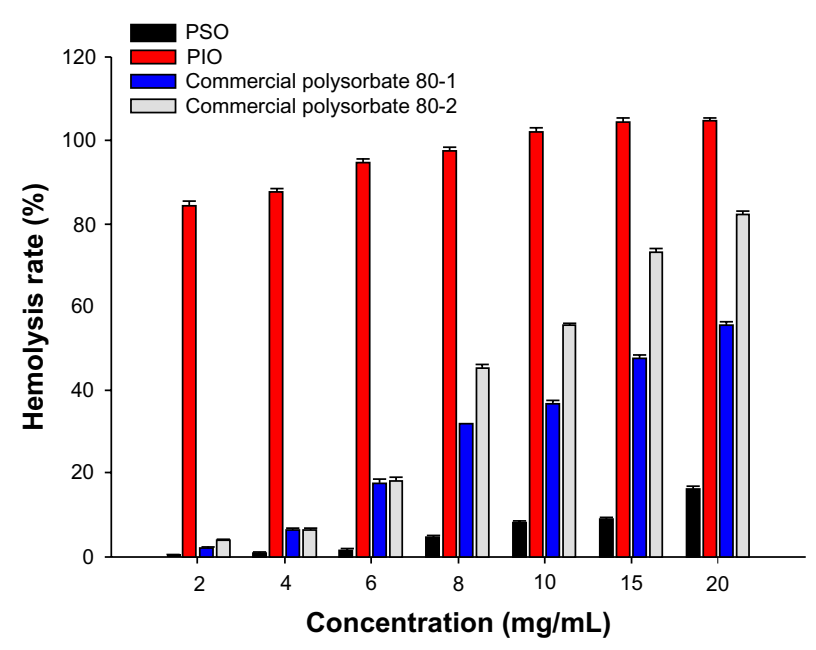

Figure 6 Hemolysis rate of PSO and PIO $(n=3)$.

Abbreviations: PSO, polyoxyethylene sorbitol oleate; PIO, polyoxyethylene isosorbide. 


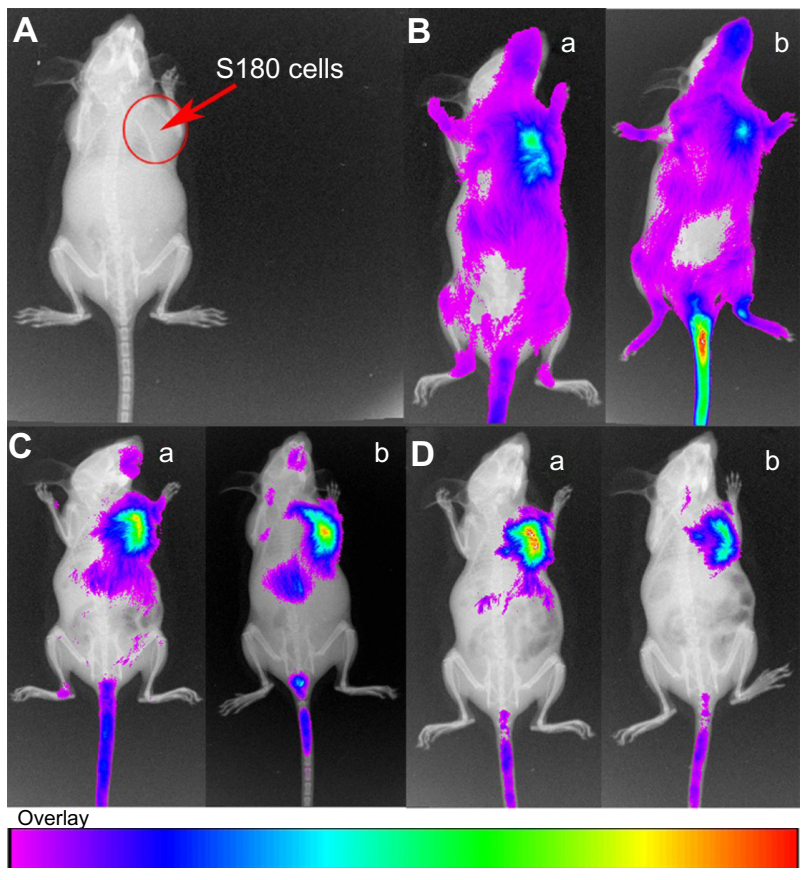

Figure 7 In vivo fluorescence imaging of SI80 tumor-bearing mice after intravenous administration of saline as the blank control. (A) DiR-loaded PSO (a) and DiR-loaded commercial polysorbate 80 (b) at 30 minutes (B), 2 hours (C), and 12 hours (D). Abbreviations: PSO, polyoxyethylene sorbitol oleate; DiR, I, I'-dioctadecyl$3,3,3^{\prime}, 3^{\prime}$-tetramethylindotricarbocyanine iodide.

via the enhanced permeation and retention effect as a result of its low particle size.

\section{Critical attributes of PSO}

Critical micelle concentration and entrapment efficiency

The critical micelle concentration of PSO was obtained using the fluorescent probe method, and the results are shown in Figure 8 . It was found that the critical micelle concentration of PSO $\left(0.462 \times 10^{-5} \mathrm{~mol} / \mathrm{L}\right)$ was not significantly different

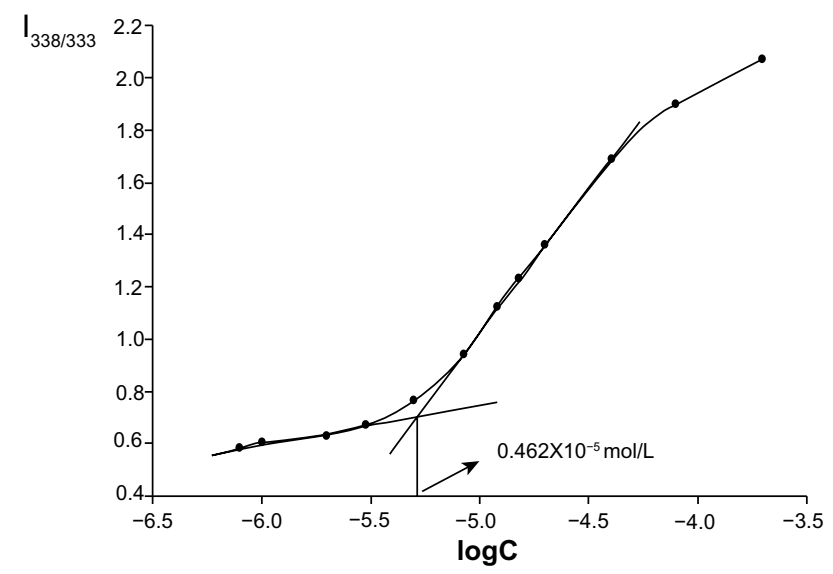

Figure 8 Critical micelle concentration for PSO. Abbreviation: PSO, polyoxyethylene sorbitol oleate. from that of commercial polysorbate 80. The EE values for docetaxel are shown in Table 3. The EE of PSO was significantly higher than that of commercial polysorbate 80 because of the presence of PIO in commercial polysorbate 80 (Figure 3C and D) which forms diesters with two fatty acid chains. The resulting diesters are not amphipathic and therefore cannot form micelles. Therefore, the effective ingredients in commercial polysorbate 80 were lower than those of PSO.

\section{Pseudoallergy triggered by PSO, PIO, and commercial polysorbate 80}

The symptoms and grades of pseudoallergy triggered by PSO, $\mathrm{PIO}$, and commercial polysorbate 80 in guinea pigs are shown in Table 4 . The in vivo study yielded results consistent with those of the in vitro $\beta$-hexosaminidase release experiment. Since the allergic reaction triggered by PNS occurs on the first administration, we dosed the study products without a presensitization period. The PSO group showed relatively mild symptoms compared with the negative control group. However, more severe symptoms, including dyspnea, wheezing rales, micturition, and defecation, were observed in the $\mathrm{PIO}$ and commercial polysorbate 80 groups, indicating that $\mathrm{PIO}$ and products containing PIO could trigger more serious pseudoallergy than PSO.

\section{Mechanism of PNS-triggered mast cell degranulation}

Mast cell degranulation and activation of the complement system are reported as two distinguishing features of pseudoallergy in the early literature. ${ }^{29,30}$ In the present study, we attempted to determine how PNS translocated into mast cells and led to degranulation and histamine release.

\section{Inhibitory effect of BAC on PNS-induced $\beta$-hexosaminidase release}

It has been reported that BAC can selectively inhibit the release of histamine induced by compound 48/80 and other

Table 3 Entrapment efficiency for different samples $(n=3)$

\begin{tabular}{lll}
\hline m (solubilizer): & \multicolumn{2}{l}{ Entrapment efficiency } \\
\cline { 2 - 3 } m (DTX) & PSO & $\begin{array}{l}\text { Commercial } \\
\text { polysorbate 80 }\end{array}$ \\
\hline $5: 1$ & $27.53 \% \pm 2.3 \%$ & $25.10 \% \pm 3.0 \%$ \\
$15: 1$ & $89.28 \% \pm 3.7 \%$ & $68.92 \% \pm 1.9 \% *$ \\
$25: 1$ & $97.62 \% \pm 1.5 \%$ & $87.95 \% \pm 1.1 \% *$ \\
\hline
\end{tabular}

Note: $* P<0.05$ versus PSO.

Abbreviations: m, moles; DTX, docetaxel; PSO, polyoxyethylene sorbitol oleate. 
Table 4 In vivo symptoms of pseudoallergy of different samples $(n=4)$

\begin{tabular}{lll}
\hline Samples & Reaction symptoms & Level \\
\hline Negative & Normal & - \\
Positive & Death & ++++ \\
PSO & Slight shivering & + \\
PIO & Acute shivering, piloerection, & +++ \\
& wheezing rales, dyspnea & \\
Commercial & Continuous shivering, & ++ \\
polysorbate 80-I & micturition, defecation & \\
Commercial & Continuous shivering, micturition, & ++ \\
polysorbate 80-2 & defecation, sneezing, cough & \\
\hline
\end{tabular}

Abbreviations: PSO, polyoxyethylene sorbitol oleate; PIO, polyoxyethylene isosorbide oleate.

polyamines ${ }^{24}$ so we investigated if BAC has a similar inhibitory action on PNS-mediated responses.

According to the data shown in Figure 9A, BAC had a negative impact on $\beta$-hexosaminidase release triggered by PSO, PIO, and commercial polysorbate 80 . As the concentration of BAC increased, the $\beta$-hexosaminidase release rate decreased dramatically in each group. This result indicates that PNS may have a molecule that follows a similar pseudoallergy pathway as $48 / 80$ to promote degranulation followed by release of histamine, and that the pseudoallergic reaction induced by PNS occurs via a polyamine receptor-mediated pathway.

\section{Temperature-dependence and energy-dependence} of PNS-induced $\beta$-hexosaminidase release

It is well known that receptor-mediated endocytosis is both temperature-dependent ${ }^{31}$ and energy-dependent. ${ }^{26}$ Therefore, in this study, we investigated whether these factors have an inhibitory effect on $\beta$-hexosaminidase release triggered by PNS.

Figure $9 \mathrm{~B}$ and $\mathrm{C}$ shows that mast cell degranulation was inhibited at a low temperature $\left(4^{\circ} \mathrm{C}\right)$. Degranulation in all groups was intensively reduced at a concentration of $1 \%$. Research has demonstrated that temperature can influence the initial steps in receptor-mediated endocytosis. ${ }^{31}$ In contrast, this temperature-dependent inhibition was not seen in the cytolysis group (Triton X-100), suggesting that the effect was not caused by mast cell lysis. Figure $9 \mathrm{C}$ clearly demonstrates that mast cell degranulation is an energy-dependent process. Significantly low $\beta$-hexosaminidase was detected after incubation with PSO, PIO, and commercial polysorbate 80 in the adenosine triphosphate-exhausted groups pretreated with 2-deoxy-D-glucose. On the basis of these data, we assume that PNS may enter mast cells via polyamine receptor-mediated endocytosis in a temperature-dependent and energy-dependent manner to induce mast cell degranulation followed by release of histamine and $\beta$-hexosaminidase and concomitant pseudoallergy.

Inclusion of PNS has been found to increase the water solubility of hydrophobic anticancer drugs, prevent drugs from degradation and inactivation, and reduce their side effects and toxicity. In the present work, a new PNS based on polysorbate 80 , ie, PSO, was successfully developed for the purpose of creating a more hypoallergenic and hypohemolytic nanocarrier for docetaxel. Taxotere ${ }^{\circledR}$, a widely used docetaxel preparation developed by Sanofi-Aventis, uses polysorbate 80 as a solubilizer. However, there is now substantial evidence
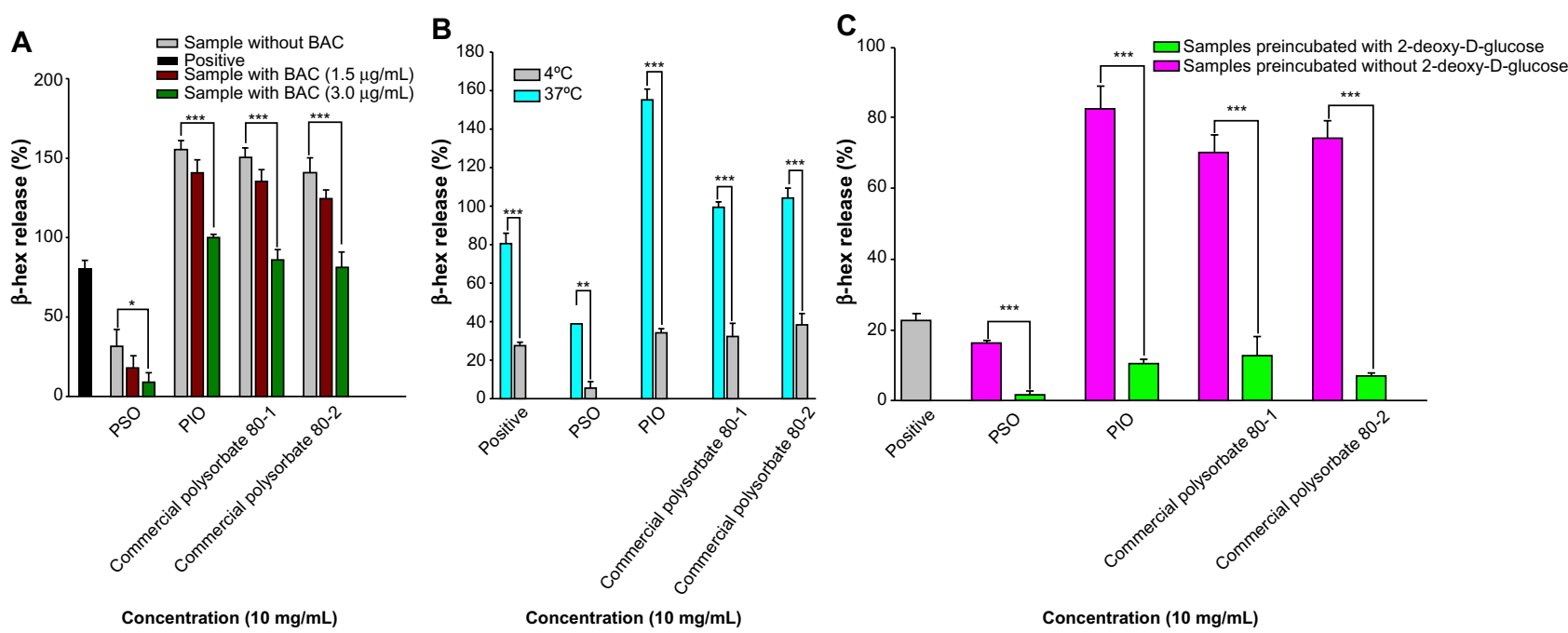

Figure 9 (A) PNS-induced $\beta$-hexosaminidase release assay in the presence of $B A C$. The data are shown as the mean \pm standard deviation ( $n=5$ ). $* P<0.05$, $* * * P<0.00$ I. (B) PNS-induced $\beta$-hexosaminidase release at $4^{\circ} \mathrm{C}$ and $37^{\circ} \mathrm{C}$. The data are shown as the mean \pm standard deviation ( $\mathrm{n}=5$ ). $* * P<0.0 \mathrm{I}, * * * P<0.00 \mathrm{I}$. (C) $\mathrm{PNS}$-induced $\beta$-hexosaminidase release assay with and without adenosine triphosphate. The data are shown as the mean \pm standard deviation $(\mathrm{n}=5)$. $* * * P<0.00 \mathrm{I}$.

Abbreviations: BAC, benzalkonium chloride; PSO, polyoxyethylene sorbitol oleate; PIO, polyoxyethylene isosorbide; PNS, polyoxyethylene nonionic surfactant. 
that polysorbate 80 can induce severe pseudoallergy and hemolysis in patients, even when receiving this product for the first time. PSO could be an alternative to polysorbate 80 for delivery of docetaxel because of its safety and effectiveness. The molecular structure of PSO was also accurately determined (Figure 3A).

It was shown early on that the histamine release induced by compound $48 / 80$ is a two-stage process, ie, primary energy-requiring transport of histamine-containing granules to the outside of the mast cell and a secondary nonenergyrequiring physicochemical process or extracellular cation exchange between histamine and cations (mainly sodium) in the shed granules. ${ }^{32}$ Sensitization without a presensitization period is the major difference between pseudoallergy and normal allergy, indicating that pseudoallergy is not an immunoglobulin E-mediated allergic reaction. Therefore, it is crucial to determine how the allergen (eg, PNS) enters mast cells to induce escape of granules.

The results of the present work suggest a novel structure based hypothesis for an effective explanation of PNS-mediated pseudoallergy. Different polysorbate 80 matrices including sorbitol, sorbitan, and isosorbide were tested, and it was found that these matrices have different actions in terms of pseudoallergy. PSO had the lowest degranulation rate while PIO had the highest degranulation rate. To the best of our knowledge, the toxicity of excipients in the isosorbide matrix has not been reported elsewhere. Further, the mechanism of mast cell degranulation was investigated, and it was concluded that polyamine receptor-mediated endocytosis might be involved in the uptake of PNS into mast cells. The hemolysis rate, EE, and critical micelle concentration were also investigated, and an in vivo study of pseudoallergy was performed to determine if the critical attributes of PSO are similar to those of commercial products and therefore suitable for clinical research.

This study has helped to develop a new PSO with lower toxicity, and also suggests a promising way to improve the current technology for manufacturing other types of PNS. Moreover, our results with regard to the hemolysis and pseudoallergy reactions associated with PIO can be used in the design and synthesis of novel nanomaterials for drug delivery systems and improve the standards for PNS in the pharmacopeia. Our team is currently trying to modify this safer nanocarrier molecule (PSO) with other functional groups.

\section{Conclusion}

In this study, hypoallergenic and hypohemolytic amphiphilic PSO was successfully developed. This nanocarrier had excellent $\mathrm{EE}$ for docetaxel and shows improved tumor-targeting ability. The most important advantage of this design is that PSO has a lower risk of inducing pseudoallergic and hemolytic reactions compared with commercial polysorbate 80 . PIO, a component of polysorbate 80 as defined by the United States Pharmacopeia 35-National Formulary 30, may be the culprit responsible for pseudoallergy induced by polysorbate 80 . The structure of PIO can guide the synthesis of novel carriers to avoid hemolysis and pseudoallergic reactions. The results of our mechanistic studies suggest that the initial step in the pseudoallergic reaction induced by PNS may involve polyamine receptor-mediated endocytosis.

\section{Acknowledgments}

This work was financially supported by the China National Key Hi-Tech Innovation Project for the R\&D of Novel Drugs (2011ZX11501) and the Fundamental Research Funds for the Central Universities (2011ZX09501-001-06). The authors are grateful to their cooperating partners from Will Chemical Co, Ltd, for assisting with the synthesis of PSO and PIO.

\section{Disclosure}

The authors report no conflicts of interest in this work.

\section{References}

1. Li YP, Xiao K, Luo JT, Lee J, Pan SR, Lam KK. A novel size-tunable nanocarrier system for targeted anticancer drug delivery. $J$ Control Release. 2010;144(3):314-323.

2. Karve S, Werner ME, Sukumar R, et al. Revival of the abandoned therapeutic wortmannin by nanoparticle drug delivery. Proc Natl Acad Sci U S A. 2012;109(21):8230-8235.

3. Wang AZ, Langer R, Farokhzad OC. Nanoparticle delivery cancer drugs. Annu Rev Med. 2012;63:185-198.

4. Kim CK, Cho YJ, Gao ZG. Preparation and evaluation of biphenyl dimethyl dicarboxylate microemulsions for oral delivery. J Control Release. 2001;70(1-2):149-155.

5. Trotta M, Gallarate M, Pattarino F, Morel S. Emulsions containing partially water-miscible solvents for the preparation of drug nanosuspensions. J Control Release. 2001;76(1):119-128.

6. Wang YJ, Wang C, Gong CY, et al. Polysorbate 80 coated poly ( $\varepsilon$-caprolactone)-poly (ethylene glycol)-poly ( $\varepsilon$-caprolactone) micelles for paclitaxel delivery. Int J Pharm. 2012;434:1-8.

7. United States Pharmacopeia 35-National Formulary 30. Rockville, MD: United States Pharmacopeia. Available from: http://www.usp.org/ usp-nf. Accessed April 1, 2014.

8. Norrie SF, Sporns P. Investigating the molecular heterogeneity of polysorbate emulsifiers by MALDI-TOF MS. J Agric Food Chem. 2001;49(7):3335-3340.

9. Szebeni J, Muggia FM, Alving CR. Complement activation by cremophor EL as a possible contributor to hypersensitivity to paclitaxel: an in vitro study. J Natl Cancer Inst. 1998;90(4):300-306.

10. Qiu SD, Liu ZH, Hou L, et al. Complement activation associated with polysorbate 80 in beagle dogs. Int Immunopharmacol. 2011;15(1): 144-149.

11. Szebeni J. Complement activation-related pseudoallergy: a new class of drug-induced acute immune toxicity. Toxicology. 2005;216(2):106-121.

12. Descotes J, Payen C, Vial T. Pseudo-allergic drug reactions with special reference to direct histamine release. Perspect Exp Clin Immunotoxicol. 2007;1:40-49. 
13. Ha E, Wang W, Wang YJ. Peroxide formation in polysorbate 80 and protein stability. J Pharm Sci. 2002;91(10):2252-2264.

14. Bommer J, Wilhelms OH, Barth HP, Schindele H, Ritz E. Anaphylactoid reactions in dialysis patients: role of ethylene-oxide. Lancet. 1985;326(8469):1382-1385.

15. Naal RM, Tabb J, Holowka D, Baird B. In situ measurement of degranulation as a biosensor based on RBL-2H3 mast cells. Biosens Bioelectron. 2004;20(4):791-796.

16. WuelfingWP, Kosuda K, Templeton AC, Harman A, Mowery MD, Reed RA. Polysorbate $80 \mathrm{UV} / \mathrm{vis}$ spectral and chromatographic characteristics-defining boundary conditions for use of the surfactant in dissolution analysis. J Pharm Biomed Anal. 2006;41(3):774-782.

17. Ayorinde FO, Gelain SV, Johnson JH, Wan JLW. Analysis of some commercial polysorbate formulations using matrix-assisted laser desorption/ionization time-of-flight mass spectrometry. Rapid Commun Mass Spectrom. 2000;14(22):2116-2124.

18. Xiong YR, Jiang WW, Shen Y, et al. A poly ( $\gamma$, L-glutamic acid)citric acid based nanoconjugate for cisplatin delivery. Biomaterials. 2012;33(29):7182-7193.

19. Natsume H, Iwata S, Ohtake K, et al. Screening of cationic compounds as an absorption enhancer for nasal drug delivery. Int J Pharm. 1999; 185(1):1-12.

20. Su ZG, Shi YP, Xiao YY, et al. Effect of octreotide surface density on receptor-mediated endocytosis in vitro and anticancer efficacy of modified nanocarrier in vivo after optimization. Int J Pharm. 2013;447(1): 281-292.

21. Jiang TY, Zhang ZH, Zhang YL, et al. Dual-functional liposomes based on $\mathrm{pH}$-responsive cell-penetrating peptideand hyaluronic acid for tumor-targeted anticancer drug delivery. Biomaterials. 2012;33(36): 9246-9258.

22. Aguiar J, Carpena P, Molina-Bolivar JA, Ruiz CC. On the determination of the critical micelle concentration by the pyrene $1: 3$ ratio method. J Colloid Interface Sci. 2003;258(1):116-122.
23. Su M, Zhao MM, Luo YF, et al. Evaluation of the efficacy, toxicity and safety of vinorelbine incorporated in a lipid emulsion. Int J Pharm. 2011;411(1):188-196.

24. Read GW, Kiefer EF. Benzalkonium chloride: selective inhibitor of histamine release induced by compound 48/80 and other polyamines. J Pharmacol Exp Ther. 1979;211(3):711-715.

25. Johnson A, Moran NC. Inhibition of the release of histamine from rat mast cells: the effect of cold and adrenergic drugs on release of histamine by compound 48/80. J Pharmacol Exp Ther. 1970;175(3):632-640.

26. Richard JP, Melikov K, Brooks H, Prevot P, Lebleu B, Chernomordik LV. Cellular uptake of unconjugated TAT peptide involves clathrindependent endocytosis and heparin sulfate receptors. J Biol Chem. 2005;280(15):15300-15306.

27. Hurtado PP, Lam PY, Kilgour D, et al. Use of high resolution mass spectrometry for analysis of polymeric excipients in drug delivery formulations. Anal Chem. 2012;84(20):8579-8586.

28. Gill IJ, Illum L, Farraj N, Ponti RD. Cyclodextrins as protection agents against enhancer damage in nasal delivery systems I. Assessment of effect by measurement of erythrocyte haemolysis. Eur J Pharm Sci. 1994;1(5):229-236.

29. Vo TS, Kong CS, Kim SK. Inhibitory effects of chitooligosaccharides on degranulation and cytokine generation in rat basophilic leukemia RBL-2H3 cells. Carbohydr Polym. 2011;84(1):649-655.

30. Suzuki Y, Miyatake K, Okamoto Y, Muraki E, Minami S. Influence of the chain length of chitosan on complement activation. Carbohydr Polym. 2003;54(4):465-469.

31. Goldenthal KL, Pastan I, Willingham MC. Initial steps in receptormediated endocytosis: The influence of temperature on the shape and distribution of plasma membrane clathrin-coated pits in cultured mammalian cells. Exp Cell Res. 1984;152(2):558-564.

32. Thon IL, Uvnas B. Degranulation and histamine release, two consecutive steps in the response of rat mast cells to compound 48/80. Acta Physiol Scand. 1967;71(4):303-315.
International Journal of Nanomedicine

\section{Publish your work in this journal}

The International Journal of Nanomedicine is an international, peerreviewed journal focusing on the application of nanotechnology in diagnostics, therapeutics, and drug delivery systems throughout the biomedical field. This journal is indexed on PubMed Central, MedLine, CAS, SciSearch $\AA$, Current Contents ${ }^{\circledR} /$ Clinical Medicine,

\section{Dovepress}

Journal Citation Reports/Science Edition, EMBase, Scopus and the Elsevier Bibliographic databases. The manuscript management system is completely online and includes a very quick and fair peer-review system, which is all easy to use. Visit http://www.dovepress.com/ testimonials.php to read real quotes from published authors. 